\title{
Fiber optic sensing of magnetic fields utilizing femtosecond laser sculpted microslots and long period gratings coated with Terfenol-D
}

\author{
Graham C. B. Lee*a, Tom Allsop ${ }^{\mathrm{a}}$, Changle Wang ${ }^{\mathrm{a}}$, Ron Neal ${ }^{\mathrm{b}}$, Philip Culverhouse ${ }^{\mathrm{b}}$ \\ and David J. Webb ${ }^{\mathrm{a}}$ \\ ${ }^{a}$ Aston Institute of Photonic Technologies, Aston University, Birmingham, UK \\ ${ }^{\mathrm{b} S c h o o l}$ of Computing and Mathematics, Faculty of Science and Technology, University of \\ Plymouth, UK
}

\begin{abstract}
Fiber optic sensors are fabricated for detecting static magnetic fields. The sensors consist of a UV inscribed long period grating with two 50 micron long microslots. The microslots are fabricated using the femtosecond laser based inscribe and etch technique. The microslots and the fiber surface are coated with a magnetostrictive material Terfenol-D. A spectral sensitivity of $1.15 \mathrm{pm} / \mathrm{mT}$ was measured in transmission with a working resolution of $\pm 0.2 \mathrm{mT}$ for a static magnetic field strength below $10 \mathrm{mT}$. These devices also present a different response when the spatial orientation of the fiber was adjusted relative to the magnetic field lines.
\end{abstract}

Keywords: magnetic sensing, long period gratings, femtosecond laser, etching, optical fibers

\section{INTRODUCTION}

In recent years there has been an increased interest in electromagnetic fields (EMF) and thus the sensors for measuring the fields, with applications in various areas of science and technology such as process control, electric field monitoring in medical apparatuses and the potential health risks caused by environmental exposure to artificial sources such as overhead power cables/pylons [1,2]. The conventional EMF measurement systems currently use active metallic probes which can disturb the measuring EMF and make the sensor very sensitive to electromagnetic noise. Fiber optic EMF sensors have shown great advantages with respect to the electronic devices such as a very good galvanic insulation, high sensitivity and very wide bandwidth. Many magnetic field optical sensors exploit the magnetostrictive effect, creating a strain in a ferromagnetic material in the direction of the magnetic field and producing a longitudinal strain in an optical fiber adhered to the material [3,4]. A magnetic field sensor using magnetic fluid and an optical microfiber mode interferometer has also been demonstrated [5,6]. All of these fiber optic sensors are non-vectorial and have relatively low sensitivity to static magnetic field strengths.

In this paper we demonstrate a fiber optic magnetic field sensor based on a long period grating (LPG) and two microslots enabling a relatively high spectral sensitivity $(1.15 \mathrm{pm} / \mathrm{mT})$ to a low magnetic field strength (of less than $10 \mathrm{mT})$ with a resolution of $60 \mu \mathrm{T}$ and the potential for vectorial sensing. These sensors are based on a UV inscribed LPG with two 50 $\mu \mathrm{m}$ long microslots parallel to the fiber core longitudinal axis, with sputtering technology used to adhere a magnetostrictive material - Terfenol-D to the optical fiber and the microslots.

\section{DEVICE FABRICATION AND CHRACTERISATION}

\subsection{Device design \& fabrication}

The first step in fabricating the magnetic sensor begins with the inscription of the LPG in hydrogenated standard single mode fiber (Corning SMF-28e). The LPG was inscribed with a $244 \mathrm{~nm}$ UV laser (Coherent Sabre Fred) with an average laser power of $100 \mathrm{~mW}$ using the point-by-point inscription method [7]. The LPG was $30 \mathrm{~mm}$ in length with a grating period of $430 \mu \mathrm{m}$. The in-fiber microslots were fabricated using the femtosecond laser based inscribe and etch technique $[8,9]$. The inscription was performed using an Amplitude Systèmes s-Pulse HP femtosecond laser that produces sub 500

*g.lee3@aston.ac.uk; phone 44121204 3524; www.aston.ac.uk

24th International Conference on Optical Fibre Sensors, edited by Hypolito José Kalinowski,

José Luís Fabris, Wojtek J. Bock, Proc. of SPIE Vol. 9634, 96340K · () 2015 SPIE

CCC code: $0277-786 \mathrm{X} / 15 / \$ 18 \cdot$ doi: $10.1117 / 12.2194325$

Proc. of SPIE Vol. 9634 96340K-1 
fs laser pulses at a center wavelength of $1026 \mathrm{~nm}$. Laser inscription was carried out under a 100x objective (Mititoyo MPlan Apo NIR Series) with a numerical aperture of 0.5. The approximate FWHM of the laser spot size incident on the samples was $1.5 \mu \mathrm{m}$. Details on the mounting configuration and method for inscribing each microslot can be found in the following reference [9]. Two $50 \mu \mathrm{m}$ microslots separated by $70 \mu \mathrm{m}$, with an offset of $28 \mu \mathrm{m}$ from the core edge were inscribed in the mid position of the LPG with a pulse energy of $350 \mathrm{~nJ}$, as illustrated in Figure 1a. This distance was chosen to provide the maximum effect on the cladding modes associated with the LPG with a period of $430 \mathrm{~mm}$ and an attenuation band around $1500 \mathrm{~nm}$, which corresponds to the $\mathrm{HE}_{1,4}$ and possibly $\mathrm{HE}_{1,5}$ cladding modes for a conventional SMF [10]. After inscription the sensor was etched in a $5 \%$ hydrofluoric acid solution assisted by an ultrasonic bath for approximately 25 minutes. The device after etching is shown in Figure $1 \mathrm{~b}$ and $\mathrm{c}$. The final step to functionalize the sensor was to back fill the microslots and to apply a uniform layer of Terfenol-D on the fiber using a sputter machine and mask to achieve a coating thickness of $1 \mu \mathrm{m}[3]$.

(a)

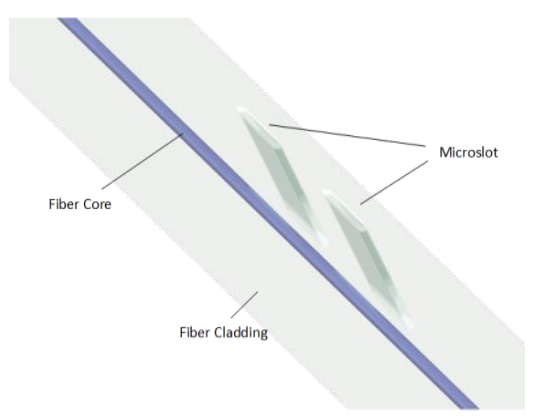

(b)

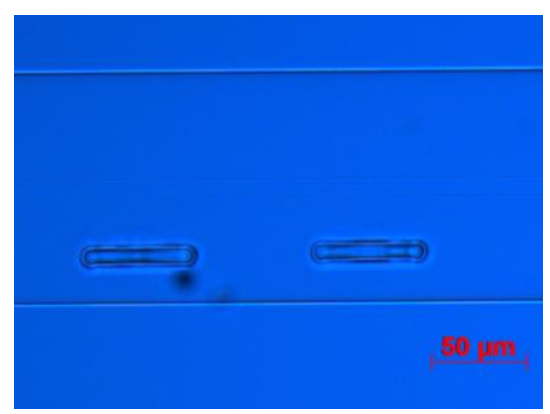

(c)

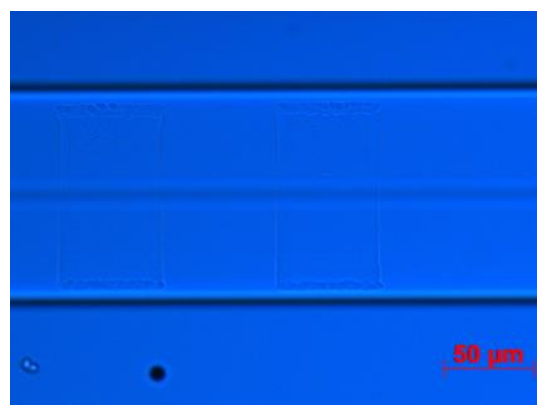

Figure 1. (a) Schematic of the magnetic optical fiber design - femtosecond laser inscribed and etched microslots positioned at the midpoint of the UV inscribed LPG. Image of the in-fiber microslots after femtosecond laser inscription and HF treatment - (b) top view focused at the center of the microslots and (c) side view showing the microslots passing through the whole fiber.

\subsection{Device characterization}

The magnetic sensor was subjected to a range of magnetic field strengths and interrogated in transmission as illustrated in Figure 2a. A broadband light source containing a series of ELEDs (Agilent 83437A, 1260nm to 1690nm) passed through a broadband motorized linear polarizer (PAT8000B), a manual polarization controller and polarimeter prior to illuminating the magnetic sensor. The transmission spectrum was observed using an OSA (Agilent 86142B). With reference to Figure 3a, magnetic fields from a ring magnet and a horseshoe magnet were used to test the fiber sensor response. The strength of each magnet was measured using a Gauss meter. The thermal sensitivity of the device was tested using a Peltier heater. The temperature sensitivity of the magnetic sensor was characterized between $20{ }^{\circ} \mathrm{C}-60{ }^{\circ} \mathrm{C}$ and calculated using the change in centroid wavelength. A linear thermal sensitivity of $12.6 \mathrm{pm} /{ }^{\circ} \mathrm{C}$ and $16.2 \mathrm{pm} /{ }^{\circ} \mathrm{C}$ was observed for the modes in the $1435 \mathrm{~nm}$ and $1550 \mathrm{~nm}$ regions respectively.

(a)

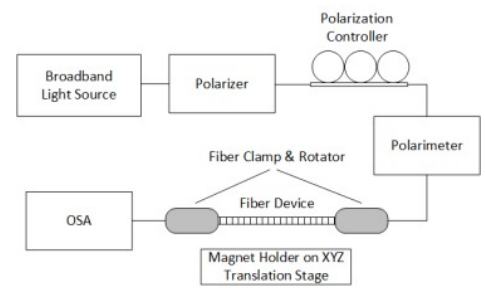

(b)

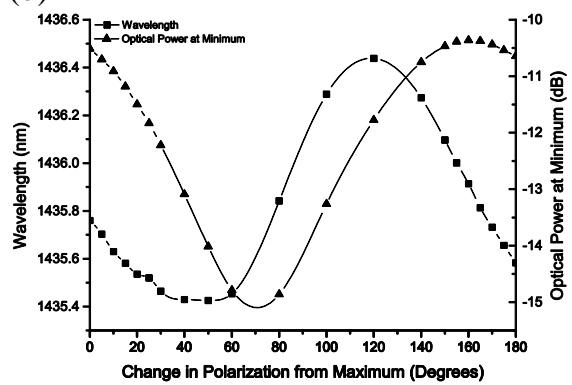

(c)

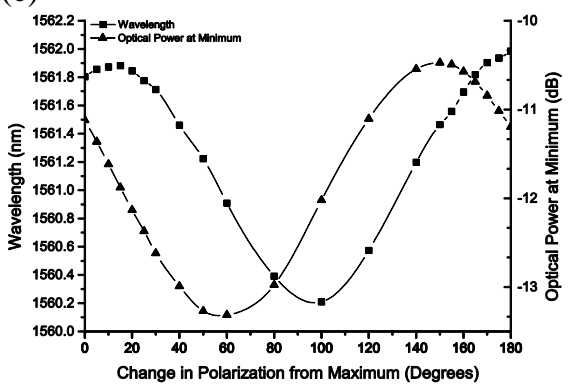

Figure 2. (a) Device characterization measurement configuration. Variation in the centroid wavelength and the effective optical strength of the transmission spectra, as a function of polarization. Two microslot device polarization responses in the (b) $1430 \mathrm{~nm}$ and (c) $1550 \mathrm{~nm}$ region. 
The polarization dependence of the two strongest modes in the $1430 \mathrm{~nm}$ and $1550 \mathrm{~nm}$ regions were studied. Given that the microslots occupy one plane through the optical fiber, there is a $90^{\circ}$ separation between one maximum to the following minimum.

\section{RESULTS AND DISCUSSION}

Two magnets were used in the following tests - a $6.4 \mathrm{~kg}$ pull ring magnet and a $4.5 \mathrm{~kg}$ pull horseshoe magnet. The ring magnet was used to examine the sensitivity of the magnetic fiber sensor in small magnetic fields $(<10 \mathrm{mT})$. The horseshoe magnet was used to evaluate the sensitivity of the magnetic sensor in much larger magnetic fields $(>10 \mathrm{mT})$ and to illustrate the potential of using the fiber device as a vectorial sensor. With the fiber sensor positioned in the hole of the ring magnet, the magnet was translated over and away from the microslots and thus subjected the fiber sensor to small magnetic fields. Figure 3a shows the magnet and optical fiber configuration. The spectral response of the fiber sensor at each magnetic field strength was recorded and a centroid peak-fitting algorithm was used to track the wavelength resonance.

(a)

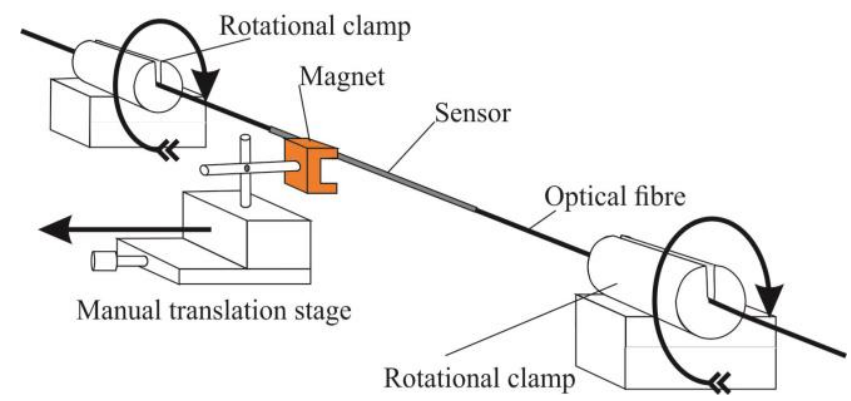

(b)

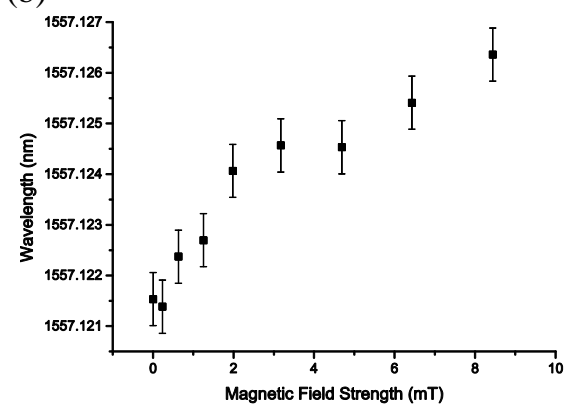

Figure 3. (a) Magnet and fiber sensor arrangement - diagram shows a magnet mounted to a 3D translation stage and the fiber sensor held in rotational clamps. (b) Variation in the centroid wavelength (1550 $\mathrm{nm}$ region) in response to small magnetic field strengths generated by a $6.4 \mathrm{~kg}$ pull ring magnet translated across the fiber sensor.

(a)

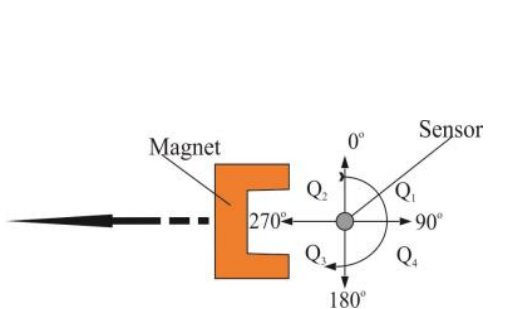

(b)

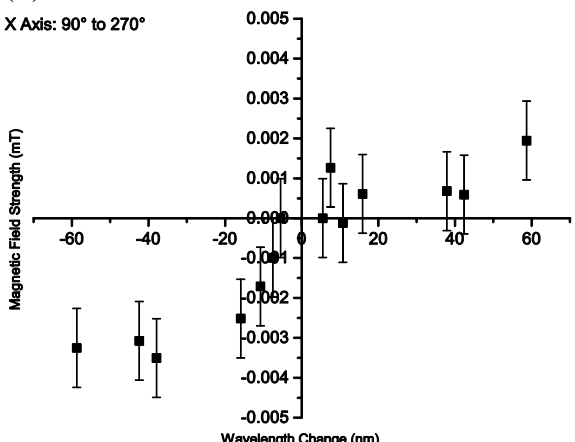

(c)

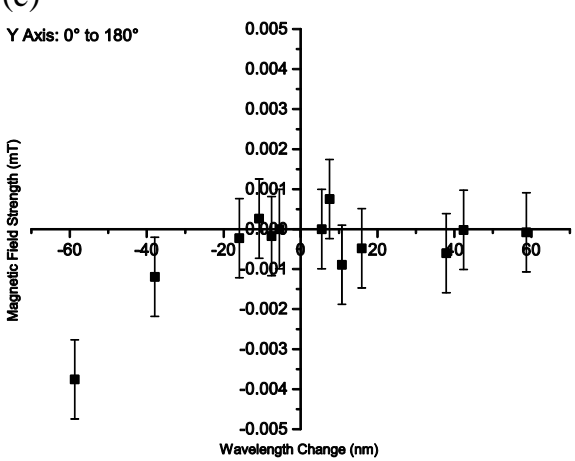

Figure 4. (a) Horseshoe magnet relative to the magnetic fiber sensor $\mathrm{X}$ and $\mathrm{Y}$ axis. Wavelength variation in response to strong magnetic field strengths in the (b) $\mathrm{X}$ axis $90^{\circ}$ to $270^{\circ}$ and (c) $\mathrm{Y}$ axis $0^{\circ}$ to $180^{\circ}$.

The sensor's resolution was determined by using the procedure in [11] providing a magnetic field strength resolution of $\sim 60 \mu \mathrm{T}$ over the range of a few $\mathrm{mT}$ and the results are shown in Figure $3 \mathrm{~b}$. A spectral sensitivity of $1.15 \mathrm{pm} / \mathrm{mT}$ was measured in transmission with a working resolution of $\pm 0.2 \mathrm{mT}$ for a static magnetic field strength below $10 \mathrm{mT}$. These devices have a spectral temperature sensitivity of $12.6 \mathrm{pm} /{ }^{\circ} \mathrm{C}$ and $16.2 \mathrm{pm} /{ }^{\circ} \mathrm{C}$ along with an average polarization dependence of $7 \mathrm{pm} /$ degree. Cross-talk between the magnetic field strength and temperature has been calculated using the experimental data obtained and other material constants from references [12] yielding a small and insignificant value $\sim 7 \times 10^{-3} \mathrm{pm}^{\circ} \mathrm{C}^{-1} \mathrm{mT}^{-1}$. 
Using the $4.5 \mathrm{~kg}$ pull horseshoe magnet, the fiber sensor was subjected to large magnetic fields strengths. This was achieved in a similar manner to the ring magnet configuration, however, in this instance the horseshoe magnet was held at the side of the fiber. The horseshoe magnet was then translated away from the fiber to vary the strength of the magnetic field imposed on the optical sensor. With reference to Figure 4a, this test was repeated at four positions on the fiber by using the fiber rotation clamps. The wavelength variation in response to the different magnetic field strengths relative to the orientation of the fiber sensor is shown in Figure $4 \mathrm{~b}$ and $\mathrm{c}$. The overall sensitivity compared to the previous experiment is less. This is expected, due to the fact that the magnetic field direction (along the axis of the fiber) is chosen to obtain the maximum response of the Terfenol-D monoliths within the optical fiber. Here the spectral sensitivity is $0.05 \mathrm{pm} / \mathrm{mT}$, which is significantly lower than for the experimental data shown in Figure $3 \mathrm{~b}$. This can be improved by increasing the number of monoliths in the sensor. What is significant is that the wavelengths are opposite and with approximately the same magnitude in the spectral sensitivity along the $0^{\circ}$ and $180^{\circ}$ orientation, along the positive and negative $Y$ axis. Similarly the spectral response of the sensor along the $90^{\circ}$ and $270^{\circ}$ orientation, along the positive and negative $\mathrm{X}$ axis, again shows a distinctive response to those two orientations. Ideally a similar behavior to that of Figure $4 \mathrm{~b}$ would have been observed. It is suspected that the maximum response in the positive $\mathrm{X}$ direction is at a different angle, and that the different Terfenol-D monoliths are asymmetric in strength response due to the amount of material in each microslot that constitutes the monolith.

\section{CONCLUSION}

Fiber optic sensors were fabricated for the application of detecting and measuring static magnetic fields. The sensor consisted of a UV inscribed LPG with two 50 micron long microslots parallel to the fiber core longitudinal axis. The microslots were fabricated using the femtosecond laser based inscribe and etch technique, which allows full control of the size, shape and position of the microslots. The microslots and the fiber surface were coated in Terfenol-D. A spectral sensitivity of $1.15 \mathrm{pm} / \mathrm{mT}$ was measured in transmission with a working resolution of $\pm 0.2 \mathrm{mT}$ for a static magnetic field strength below $10 \mathrm{mT}$. In addition, these devices provide different responses when the spatial orientation of the fiber is changed relative to the magnetic field lines, thus showing potential of being true B-field sensors measuring magnitude and direction.

\section{REFERENCES}

[1] Stavroulakis, P., [Biological effects of electromagnetic fields] Springer, (2003).

[2] Sienkiewicz, Z., "Biological effects of electromagnetic fields," Engineering Science \& Education Journal, 7(3), 127-134 (1998).

[3] Smith, G. N., Allsop, T., Kalli, K., Koutsides, C., Neal, R., Sugden, K., Culverhouse, P., and Bennion, I., "Characterisation and performance of a Terfenol-D coated femtosecond laser inscribed optical fibre Bragg sensor with a laser ablated microslot for the detection of static magnetic fields," Optics Express, 19(1), 363-370 (2011).

[4] Silva, R. M., Chesini, G., Gouveia, C. J., Lobo Ribeiro, A. B., Frazão, O., Cordeiro, C. M. B., and Jorge, P. A. S., "Magnetic field sensor with Terfenol-D thin-film coated FBG." 8421, 84213C-84213C-4.

[5] Zheng, Y., Dong, X., Chan, C. C., Shum, P. P., and Su, H., "Optical fiber magnetic field sensor based on magnetic fluid and microfiber mode interferometer," Optics Communications, 336(0), 5-8 (2015).

[6] Deng, M., Liu, D., and Li, D., "Magnetic field sensor based on asymmetric optical fiber taper and magnetic fluid," Sensors and Actuators A: Physical, 211(0), 55-59 (2014).

[7] Hill, K. O., Malo, B., Vineberg, K. A., Bilodeau, F., Johnson, D. C., and Skinner, I., "Efficient mode conversion in telecommunication fibre using externally written gratings," Electronics Letters, 26(16), 1270-1272 (1990).

[8] Lai, Y., Zhou, K., Zhang, L., and Bennion, I., "Microchannels in conventional single-mode fibers," Opt. Lett., 31(17), 2559-2561 (2006).

[9] Lee, G. C. B., Mou, C., Zhou, K., and Sugden, K., "Optimization and characterization of femtosecond laser inscribed in-fiber microchannels for liquid sensing." Proc. SPIE 9157, 915743-915743-4 (2014).

[10] Allsop, T., Webb, D. J., and Bennion, I., "A comparison of the sensing characteristics of long period gratings written in three different types of fiber," Optical Fiber Technology, 9(4), 210-223 (2003).

[11] Hu, J., Sun, X., Agarwal, A., and Kimerling, L. C., "Design guidelines for optical resonator biochemical sensors," Journal of the Optical Society of America B, 26(5), 1032-1041 (2009).

[12] Ghosh, G., [Handbook of Thermo-Optic Coefficients of Optical Materials with Applications] Academic Press, (1998). 